\title{
LAS VARIEDADES Y SU COMPLEJIDAD CONCEPTUAL EN EL DISEÑO DE UN MODELO LINGÜÍSTICO PARA EL ESPAÑOL L2/LE
}

\author{
M. ${ }^{\mathrm{a}}$ ANTONIETA ANDIÓN HERRERO \\ Universidad Nacional de Educación a Distancia \\ maandion@,flog.uned.es
}

\section{Resumen}

En la actualidad y muy especialmente para el español, la enseñanza de la lengua a hablantes no nativos tiene un enorme protagonismo. Existe una extensa bibliografía didáctica (métodos o manuales y materiales de enseñanza de español como segunda lengua o lengua extranjera) que (de)muestra el interés de este ámbito. No hay duda, se ha avanzado mucho en teoría, técnicas y aplicación de metodología, inventarios de contenidos, saberes, destrezas, etc. No obstante, hay parcelas en las que todavía es imprescindible elevar el rigor del planteamiento de base. Uno de ellos se refiere a la construcción de un modelo lingüístico de enseñanza-aprendizaje. Es acuciante, desde hace una década, la preocupación por la presencia de la variedad lingüística en los materiales didácticos españoles de EL2/LE. Ello se manifiesta a través de una creciente frecuencia de rasgos del español de América colocados -unos con más u otros con menos acierto y concierto- en las sesiones más diversas de un manual. La búsqueda de un modelo lingüístico que represente al español, no ya como sistema estandarizado, sino como muestrario organizadamente variado de la lengua es de interés general. Para atender a esta demanda presentamos una fórmula con tres componentes. Nos detenemos especialmente en dos de ellos, la variedad preferente y las variedades periféricas. Explicamos, además, las circunstancias a las que debe atender el docente al seleccionar adecuadamente los rasgos de dichas variedades para insertarlos en su modelo lingüístico.

PALABRAS CLAVE: Enseñanza de ELE, modelo lingüístico, variedad lingüística, variedades periféricas.

\begin{abstract}
Nowadays, and specially regarding to Spanish, the importance of teaching this language to non-native speakers, has an enormous relevancy. There is a large bibliography in Didactics (methods and materials on the teaching of Spanish as second or foreign language) that demonstrates the interest in this field. There is no doubt about the improvement on theory, techniques and methodology, development of inventories on the subject matter, knowledge and skills. However, there are still areas in which is still indispensable to elevate the rigor of the basic assumptions. One of them refers to the construction of a linguistic model of teaching and learning. Since a decade ago, the preoccupation for the presence of a linguistic variation in the didactic materials in Spanish of a $\mathrm{L} 2 / \mathrm{FL}$ is pressing. This is evident in the increasing frecuency of traits of the American Spanish -with more or less accuracy and coherence- on the various sessions of a textbook. Having a linguistic model that represents the Spanish language, not as a standardized system, but as an organized collection or language samples, is of general interest. In response to this demand, we introduce a formula with three components, two of which will receive here a special attention: the preferred variation, and the peripheral variations. We also explain the circumstances to be considered by the instructor when selecting the traits of such variations in order to be introduced in the linguistic model.
\end{abstract}

KEYWORDS: Spanish as a Foreign Language, Linguistic model, Peripheral variations. 


\section{Introducción}

El ámbito de la enseñanza de la lengua tiene ahora mismo un enorme protagonismo en lo que se refiere a la actualidad de la lengua. Por doquier surgen métodos de enseñanza de español como segunda lengua o lengua extranjera, la muestra editorial es voluminosa y parece haberse alcanzado el nivel didáctico necesario en cuanto a metodología, inventarios de contenidos, saberes, destrezas... Pero quedan aspectos en los que es necesario elevar el rigor del planteamiento lingüístico. Uno de ellos se refiere a la construcción de un modelo lingüístico de enseñanza-aprendizaje. Es imperativo insoslayable la preocupación por la presencia de la variedad lingüística se hace patente desde hace una década en los materiales didácticos españoles a través de una creciente frecuencia de rasgos del español de América colocados aquí y allá, siempre con las mejores intenciones pero no todas las veces con buen criterio.

El nuevo y exhaustivamente elaborado Plan Curricular (2006) del Instituto Cervantes coloca los rasgos de las variedades en progreso secuencial y por niveles en sus inventarios. Es evidente que en España se ha alcanzado un nivel de madurez en la concepción de un modelo lingüístico plural en la competencia, aunque pueda ser más o menos estandarizado o dirigido en la actuación.

Por otra parte, la formación de profesores de EL2/LE cuenta con másteres y expertos que dedican asignatura independiente a las variedades, lo que da muestra de la importancia que tiene el ámbito dialectológico y sociolingüístico en la profesionalización de los docentes. Como contribución a ello está especialmente dedicado este trabajo a formar un criterio científico a la hora de componer el modelo destinado a las aulas. Entendemos que la mejor forma de presentar este modelo es como una fórmula con tres componentes, que el profesor deberá seleccionar adecuándose a condicionantes de su particular situación docente. Situación que vendrá definida por el contexto real de docencia (lugar/comunidad) y los intereses-expectativas de los aprendices. Iremos construyéndolo paso a paso.

\section{El modelo lingüístico del EL2/LE}

Si pretendemos construir un corpus lingüístico para la enseñanza del español, ya sea segunda lengua o extranjera, asumiendo la bipolar realidad de nuestra lengua -es decir, teniendo en cuenta su unidad como sistema reducido estandarizado válido para las normas cultas del mundo hispánico y, por otra parte, la complejidad de la natural variación de una lengua muy extensa demográfica y geográficamente-, es conveniente presentar una fórmula con exponentes flexibles que permitan adaptar el modelo respecto de la situación a la que se aplique. Esta fórmula respondería a la siguiente ecuación sumatoria:

EL2/LE = ESPAÑOL ESTÁNDAR + variedad preferente + variedades periféricas 
La fórmula quedaría mejor representada si la viéramos en otra dimensión figurativa, en las que preferimos mostrar de forma circular y cuasi superpuestos el estándar y la variedad preferente por su naturaleza envolvente e interrelacionadas; mientras que las variedades periféricas quedan como inserciones particulares y distribuidas.

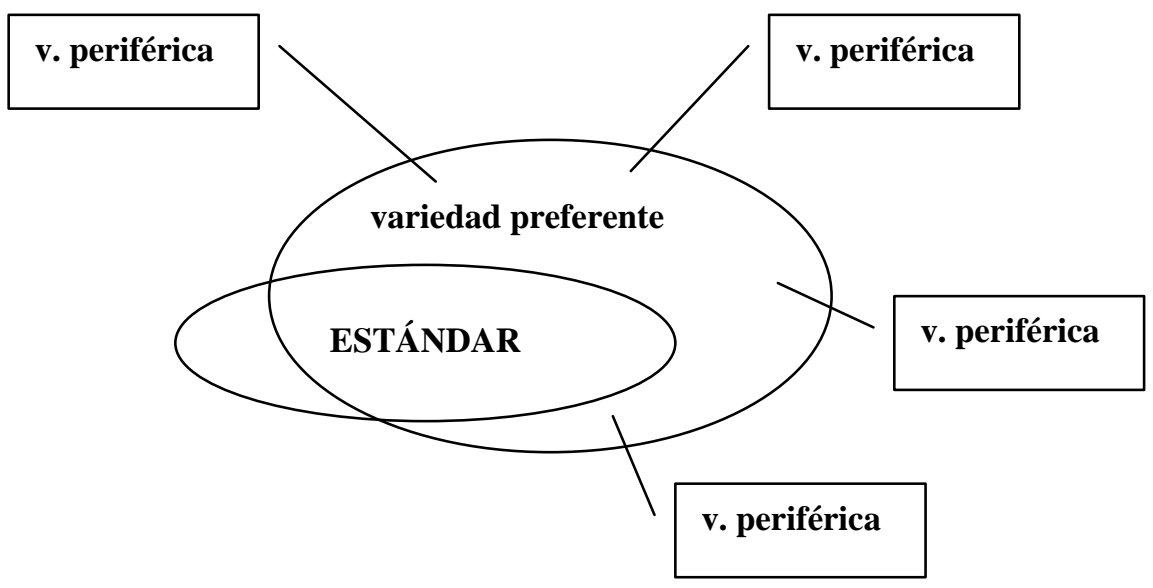

Para explicar la propia fórmula debemos antes tener claros los conceptos implicados: estándar, variedad preferente y variedad periférica. Pasemos a ello.

El concepto de estándar que manejamos, ya lo hemos definido y explicado en otro trabajo (Andión, 2008):

Lengua estándar o general:

Modelo lingüístico que cumple determinados requisitos: reunir las herramientas descriptivas y de uso para la producción y comprensión de cualquier enunciado, ser estable, accesible para sus hablantes, contar con una tradición... Los elementos lingüísticos que lo constituyen deben ser troncales o comunes a sus hablantes (Principio de comunidad) y con un grado de independencia más o menos alto de contextos específicos (Principio de neutralidad).

Es decir, en palabras de Moreno Fernández (1998: 350), el estándar o lengua general es la "variedad lingüística de una comunidad que no está marcada ni dialectal, ni sociolingüística, ni estilísticamente". Andión (2008) considera familiarmente que el estándar "representa el tronco, la médula, el meollo o la corola de la lengua". Si se nos permite la comparación, el estándar es el esqueleto de la lengua, en el que todos se ven representados, pero nadie de manera exclusiva.

No pueden faltar como primer componente del modelo lingüístico de enseñanza del español los rasgos lingüísticos que constituyen el español estándar, en cuya selección actúan criterios de comunidad (lo compartido por la mayoría de los hablantes de las 
variedades de esa lengua; por ejemplo: las formas de tratamiento tú, usted y ustedes $^{1}$ ) y de neutralidad (que no hace depender la presencia de un rasgo de un contexto casi exclusivo ${ }^{2}$ ).

Entendida la presencia del primer exponente de la fórmula

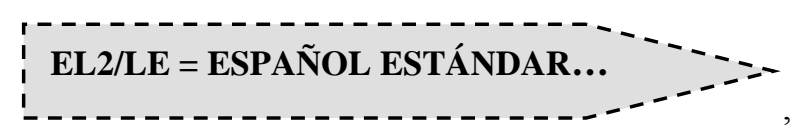

añadimos un segundo elemento, que a manera de músculo va a complementar y ampliar el estándar:

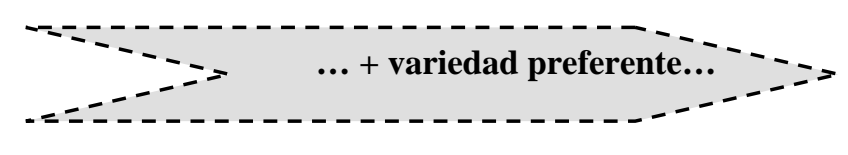

elemento que, imprescindiblemente, mostrará sus rasgos normativos. Es decir, los rasgos que considerándose correctos por una comunidad hispánica no necesariamente son comunes a todos los hispanohablantes, sino modélicos para la variedad de la lengua que queremos representar.

A estas alturas de la fórmula debemos aclarar que ya hemos de decidir la variedad que actuará como preferente o central respecto de las restantes variedades de la lengua ${ }^{3}$. Entonces, definimos la variedad como el conjunto de rasgos lingüísticos propios de una comunidad con validez geográfica determinada y que marcan el acento de sus hablantes. La variedad preferente es aquel geolecto ${ }^{4}$ del estándar ponderado dentro del programa de un curso de EL2/LE al cual le estamos diseñando un modelo lingüístico; es la variedad que hemos decidido presentar como modelo principal para la producción de los aprendices. Su selección justifica la presencia de determinados rasgos que no están en el estándar (ej.: la forma vos si nuestra variedad preferente es el español rioplatense; o la forma vosotros si es la variedad castellana).

A la variedad preferente pertenecerán las muestras de lengua que ejemplifican los contenidos lingüísticos, la mayoría de los textos (orales y escritos) del curso, los enunciados de los ejercicios en los materiales didácticos (manuales que usemos). Para seleccionar la variedad preferente debemos atender a diferentes aspectos: la naturaleza del curso (a quién va dirigido y qué objetivos persigue), si el contexto es de lengua segunda o extranjera, si

\footnotetext{
${ }^{1}$ En nuestra opinión vos y vosotros no formarían parte del estándar por no ser ni comunes ni neutros.

${ }^{2}$ Quizás al hecho de cumplir este requisito se debe el éxito y propagación del leísmo de persona en las normas del español, pues neutraliza varias oposiciones de la lengua (directo-indirecto, femenino-masculino), pese a la reticencia normativa que continúa soportando en los círculos más puristas.

${ }^{3}$ Los conceptos de variedad preferente y periférica fueron utilizados por primera vez por la prof. ${ }^{a}$ Andión en la Conferencia plenaria del X Congreso Brasileño de Profesores de Español. Natal (Brasil), en septiembre de 2003.

${ }^{4}$ Usamos geolecto en el sentido de dialecto, sistema lingüístico con delimitación geográfica concreta que no tiene una fuerte diferenciación con otros dialectos de origen común ya que todos pertenecen a la misma lengua y pueden comunicarse entre sí.
} 
está orientado a conocimientos generales o específicos (profesionales), etc. Se pueden dar situaciones de contexto en las que la selección de la variedad preferente parece lógica, es decir, en el caso de que el curso se realice en una comunidad hispánica, es natural que la variedad preferente sea la del propio contexto lingüístico que rodea a los aprendices fuera de clase. En el caso de que estemos en un contexto donde el español no es lengua oficial ni de comunicación, entrarían en juego otros factores como la cercanía o las fronteras con una zona lingüística hispánica específica. En este contexto en que el español es lengua extranjera, se podría optar por mantenerse lo más posible en los marcos del estándar y garantizar una presencia proporcionada de todas las variedades por igual.

Aprovechamos para tratar un tema que es preocupación de muchos docentes: qué hacer cuando la variedad del profesor no coincide con la variedad preferente del curso. Creemos que hay que tener una actitud flexible y a la vez de respeto hacia las variedades, empezando por la propia. No es imprescindible que coincidan la variedad del profesor y la preferente de su curso (como tampoco lo es ser nativo de la lengua que se enseña). Lo único absolutamente necesario es conocer bien la variedad que nos disponemos a presentar como preferente al alumno.

Sin modificar drásticamente los hábitos lingüísticos propios, es natural que dentro del aula el profesor imite rasgos de la variedad preferente en el momento que los está ejemplificando ante su alumnado, pero no tiene que asumirlos de manera permanente. A su vez, también es recomendable neutralizar, dentro de una buscada naturalidad, aquellos rasgos que crean problemas de significado o no pertenecen a la norma culta del profesor y son especialmente disonantes con la variedad preferente. Cuando no tenemos la variedad preferente que enseñamos, sobre todo en un contexto de lengua extranjera, debemos buscar fuentes de input que permitan al alumno tener una exposición abundante de ella.

Establecido el corpus estándar y la variedad preferente del curso, habremos de añadir un cuarto paso en la creación del corpus lingüístico que es objeto de nuestra enseñanza: la selección de las variedades periféricas

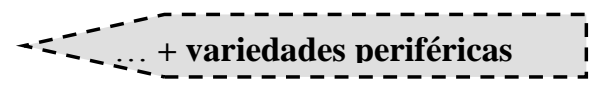

que rodean a la preferente a través de los rasgos que no comparten. Las variedades periféricas son, entonces, geolectos del estándar diferentes a la variedad preferente o central del curso. Este conocimiento de otras realizaciones de la lengua que no son el modelo concreto que se le presenta al aprendiz para su imitación productiva le compensará vacíos de información lingüística pasiva que le permitirá acceder al input de hablantes procedentes de otras zonas geográficas, y por ende, a su diversidad dialectal.

La presencia de las variedades periféricas en un curso de español lengua segunda o extranjera permite el cumplimiento varios objetivos: representar la validez del estándar para la comprensión-producción de la lengua meta en sus diferentes realizaciones geográficas, sobre todo en el nivel culto; mostrar la diversidad de realizaciones posibles para la misma lengua y completar su conocimiento real; desarrollar estrategias de comunicación (buscar 
información no explícita, desambiguar frases, preguntar por lo que no se conoce, etc.) y educar en la tolerancia y la interculturalidad.

Aunque no parezca directamente relacionado, el respeto a las variedades es un principio de educación para la convivencia, está en la base de lo que se conoce como multiculturalidad. Así lo reconoce Narbona Jiménez (2001) cuando expresa "En lo que atañe a lo idiomático, no es aplicable la frase respeto, pero no comparto, que tanto se emplea para otras normas que rigen la convivencia social. No basta con tolerar, admitir o aceptar los usos lingüísticos que no son propios, entre otras cosas, porque tampoco son ajenos -aunque no pertenezcan necesariamente al nivel de lo realizado-, desde el momento en que se sienten compartidos. De sobra se sabe que el respeto hacia algo que no se comparte tiene más de prevención y desconfianza que de neutro acatamiento"s.

\section{Selección de rasgos de las variedades periféricas}

A las variedades periféricas se les pueden aplicar dos criterios para justificar su presencia: el geográfico y el lingüístico. Si seleccionamos el primero, tendremos que tener especial cuidado para no convertir nuestro curso de EL2/LE en uno de dialectología hispánica donde abunden exhaustivas -inútiles e inaccesibles- presentaciones de cada una de las variedades periféricas. El segundo, el lingüístico, parece más pertinente y supone agrupar los rasgos de las variedades periféricas que interesan especialmente ${ }^{6}$ o de todas en diferentes niveles lingüísticos, donde fuera pertinente dentro del programa del curso. Es decir, en un curso en Ciudad de México, cuya variedad preferente es la mexicana (mesetaria), resulta más conveniente hablar de los rasgos que difieren de ella (aspiración de la -s implosiva, pérdida de las consonantes oclusivas intervocálicas, etc.) y mencionar las variedades que los tienen, antes que dedicar una lección a hablar del andaluz, otra al caribeño, etc. Es mejor para el alumno saber que el rasgo de conservación consonántica de los mexicanos del altiplano no se produce en otras variedades, sino por el contrario, que éstas eliden las consonantes y no las vocales. Resulta más rentable aprender esto que presentar monográfica y exhaustivamente cómo hablan los caribeños.

Teniendo en cuenta este principio de agrupación lingüística, queda establecer qué requisitos han de cumplir los rasgos de las variedades periféricas para pasar al corpus lingüístico de un curso. Según anota Andión (2005: 8), los rasgos deben:

1. Ser suficientemente perceptibles para el aprendiz. Identificar los rasgos no debe suponer un adiestramiento especial, más de filólogos que de usuarios de la lengua, aunque sean adoptivos.

2. Ser rentables para que merezcan la adquisición activa o pasiva. Es decir, que no conocer el rasgo pueda producir un obstáculo en la comunicación - aunque sólo sea comprensiva - de la lengua.

\footnotetext{
${ }^{5}$ Las cursivas son del propio autor.

${ }^{6}$ Dentro de las propias variedades periféricas las hay más cercanas que otras a un contexto de segunda lengua o lengua extranjera. Por ejemplo, si pensamos en un curso general de español en Porto Alegre, Estado de Rio Grande do Sul, Brasil, cuya variedad preferente fuera la del Río de la Plata, entre las variedades periféricas consideradas tendría mayor interés, por evidentes razones geográficas, la andina que la caribeña.
} 
3. Tener un área o territorio de validez y vigencia lo suficientemente amplio como para justificar que forme parte del input al que será sometido el aprendiz.

Cada rasgo debe ser insertado en el lugar que le resulte afín dentro de la programación general del curso, teniendo especial cuidado en su graduación respecto del nivel de dominio de la lengua correspondiente. Así procede el nuevo Plan curricular de Instituto Cervantes (2006) al ofrecer la inserción de rasgos de otras variedades en los inventarios lingüísticos y para ello ha recabado la participación de especialistas de todo el mundo hispánico.

\section{Las variedades periféricas en el Plan Curricular del IC}

El Plan Curricular, complejo y riguroso documento de referencia en la enseñanza del español L2/LE, ha tenido en cuenta en su presentación de contenidos la complejidad a la hora de describir la lengua común y la comunidad cultural hispánica. Estos presupuestos han sido considerados a la hora de seleccionar el material lingüístico que aparece en las Descripciones, el cual corresponde preferentemente a la norma culta de la variedad centronorte peninsular española. La selección de esta variedad está sustentada por no estar ésta en interacción con otras lenguas, por los rasgos comunes que comparte con las restantes normas cultas del mundo hispánico y por su proyección dentro del modelo estandarizado prestigioso de la lengua para la propia comunidad hispánica, a lo que habría que añadir la propia adscripción de la institución encargada de elaborar el Plan curricular. No por ello, se ignora que el español tiene la cualidad de ser una lengua que cuenta con varias normas cultas que pertenecen a diferentes localizaciones geográficas, la correspondiente a la norma centro-norte peninsular española es sólo una de ellas. De ahí que sean anotadas y comentadas especificaciones de considerable extensión en las que la norma central descrita no coincide con amplias zonas lingüísticas del mundo hispánico. Esta flexibilidad en el inventario compensa la restricción que supone describir preferentemente sólo una de las muchas variedades del español y enriquece la representación del corpus.

Las especificaciones de otras variedades del español responden a varias consideraciones. Primeramente, sus apariciones están en relación con rasgos que les sirven de referencia y que son particulares -entiéndase, casi exclusivos- de la variedad centralnorte peninsular de España y que, por lo tanto, no se comparten con otras variedades del español; lo cual recomienda anotar la diversidad de realizaciones lingüísticas válidas (gramaticales, fonéticas, nocionales, etc.), siempre atendiendo de manera preferente a la representación de la norma culta de todas las variedades. Su inserción en el inventario ha sido planificada según la propia graduación interna por niveles y responde a su relación lingüística con los contenidos descritos. En la presentación de estos rasgos siempre están anotadas las áreas geográficas de uso.

Veamos algunos ejemplos de inserción de diferentes rasgos secuenciados según sus niveles y tipo de inventarios. 
5. Los posesivos

Forma

- Formas átonas. Un poseedor. Variación de número

mi, mis, tu, tus, su, sus milibro / mis libros

- Formas tónicas y átonas. Varios posee-

4.2.3. Futuro

- destino

- la semana/la década próxima, el mes/el año/el siglo próximo

n futuro próximo/lejano

- futuro un proyecto futuro

- (ya) veremos No me comprometo a nada, ya veremos si puedo ir.

- próximamente Próximamente se celebrará un debate público entre los candidatos.

ahora mismo

Ahora mismo te lo traigo.

[Hispanoamérica] ya mism

- ya

No te preocupes, ya lo bago yo.

- a corto/a medio/a largo plazo

- a partir de ahora, desde ahora

Nociones Generales, B1-B2, Tomo II, pág. 417.
Forma

- Formas tónicas. Un poseedor. Variación de género y número mio/a/os/as, thyo/a/os/as, suyo/a/os/as

- Formas tónicas. Varios poseedores. Variación de género y número

[Hispanoamérica] Tendencia a exp sar la posesión mediante estructuras pospuestas. Posesivos pospuestos analíticos y sintéticos

el libro trivo [= tu libro] Lescuela de nosotors $[=$ nuestra ca, A1-A2, Tomo I, pág. 117. 


\section{Los fonemas y sus variantes}

5.1. Identificación y producción de los fonemas vocálicos

5.1.1. Casos de relajación de las vocales inacentuadas en pronunciación rápida y familiar

* Vocal débil, final de grupo, ante pausa lunes $>$ linn [a]s

- Penúltima vocal de una palabra esdrújula capitulo >capit [ü]lo

- Vocal pretónica en interior de palabra temeroso $>$ tem $[3 \mid$ róso

- Pronunciación de ciertas vocales dependiente de la estructura rítmica del conjunto:

- Calle de Goya (la ue» de la preposición se relaja porque va situada entre dos palabras bisílabas

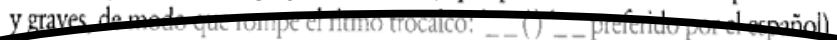

- México (altiplanicie) y regiones andinas] Debilitamiento hasta su desaparición de vocales átonas ante so consonante sorda: [p's] pues

Pronunciación y Prosodia, B1-B2, Tomo II, p. 140.

3. Conducta interaccional

3.1. Cortesía verbal atenuadora

3.1.1. Atenuación del papel del hablante o del oyente

Desplazamiento pronominal de la 2." persona

- A $3 .^{2}$ persona

- Con usted / ustedes para mostrar respeto social o distancia afectiva frente al interlocutor

¿A qué se dedica usted?

\section{sociocuiturales 2}

[Hispanoamérica, España: varieda meridionales, Canarias] Uso exclusi vo de la forma ustedes para la $2 .^{2}$ persona del plural. Ausencia de las formas vosotros / vosotras

- [Hispanoamérica] Preferencia por el iratamiento de usted ante marcadores de desconocimiento, jerarquía, tancia social, diferencias de sex
3.1.1. Atenuación del papel del hablante o del oyente

Desplazamiento pronominal de la

1." persona

- A estructuras con valor impersonal

- Con se, para minimizar la amenza a la propia imagen del hablante ¿Se puede?

¿Se puede pasar? [ = ¿Yo puedo / nosotros podemos pasar?].

- Con hay, para minimizar la fuerza ilocutiva del acto (orden o recriminación)

Hay que estudiar más [ = Tú tienes que estudiar más].

Hay que llevar corbata.

Tacticas y Estrategias Pragmáticas, A1-A2, Tomo I, pág. 265. 


\section{Productos y creaciones culturales}

3.1. Literatura y pensamiento

- Grandes autores y obras literarias de proyección intemacional

- Importancia de Cervantes y el Quijoce en la historia de la literatura universal

- Valor e impacto de los grandes personajes y obras de la literatura en la cultura y en el lenguaje populares: el caso especial del Quinote
- Grandes autares esoañoles e hisEis reteio de los grandes hitos hits

róricos en la literatura La llegadada de Colón a Americica y los

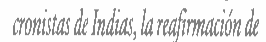
las independencias nacionders y la noveld de la tiema, la bistoria hecha relato en los Episodios Naciondes, Laperdidd delas colonidasy lagenerentción del 198, La poesía de José Martí,

Lanovela de la revoluccion mexicanan

el ciclo del dictador, la Guerta C
- Cronología de la historia de la literatura española (origenes, guandes movimientos y tendenclas): del Poema del Mio Cid a la literatura de la postmoderniCronologia de la historia de literatura en Hispanoaménca forigenes, gaardes movinimientos y yendencias): de la Crónica de Indias al post-boom de la literatura hispanoamericana 


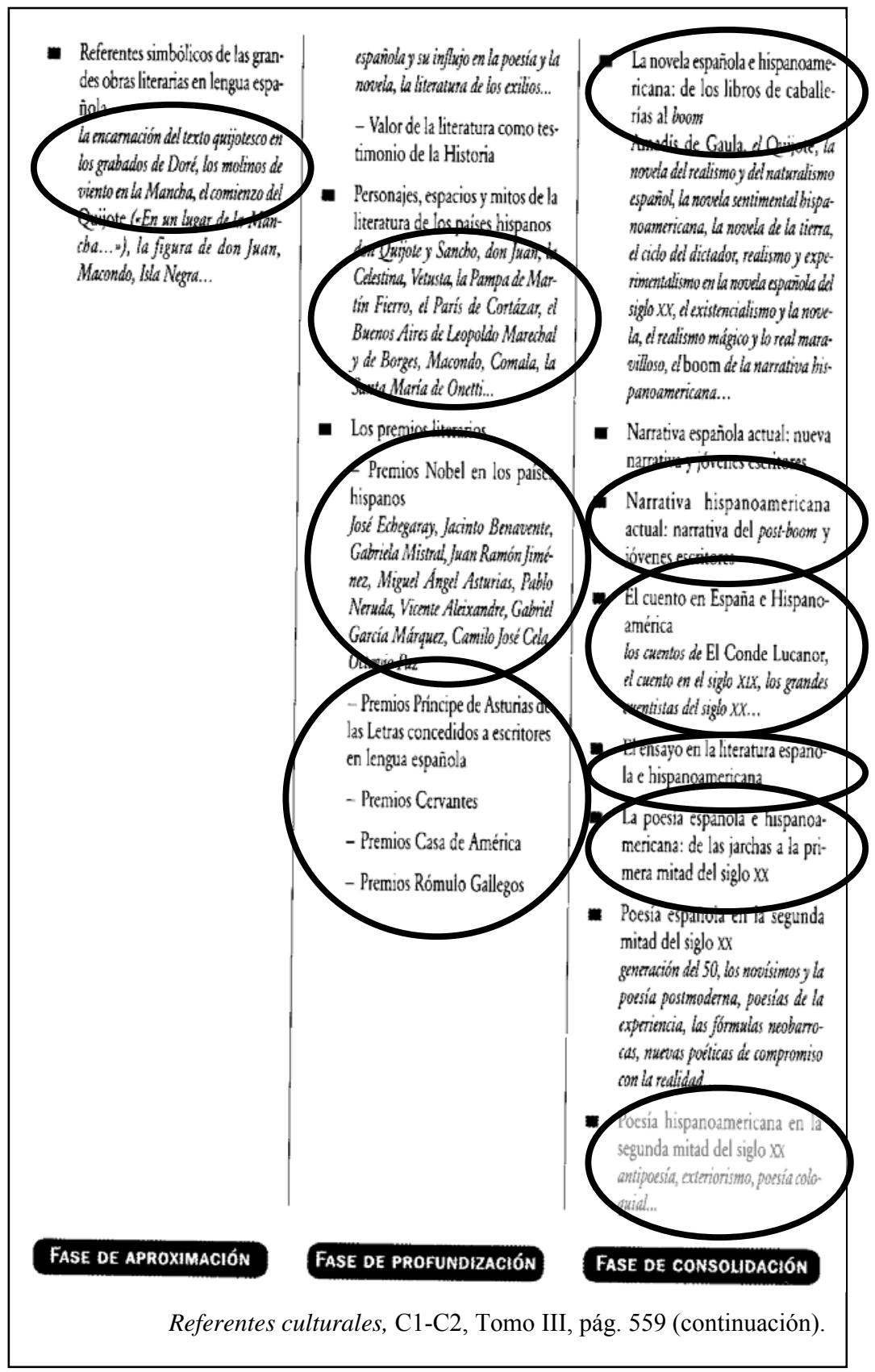


La aplicación rigurosa de los criterios antes comentados $\left(1^{\circ}, 2^{\circ}\right.$ y $\left.3^{\circ}\right)$ en esta presentación da como resultado un inventario enriquecido y plural que abarca la realidad de una lengua rentable, común y extensa, y de la diversidad cultural de la comunidad de hablantes a la que corresponde.

\section{Conclusiones}

Para el español, la enseñanza de la lengua a no nativos impone una serie de toma de decisiones imprescindibles y previas al establecimiento del corpus lingüístico objeto de enseñanza. El modelo lingüístico que se ofrezca al aprendiz de EL2/LE debe sintetizar, representar y, a la vez, hacer un muestreo de la diversidad de la lengua, sin dejar de señalar un referente claro y rentable para el alumno que le sirva para su producción en la lengua de adopción. El proceso de elaboración de ese modelo es delicado: supone aplicar con rigor conceptos como estándar, norma y variedad.

Dentro de las variedades han de establecerse grados de pertinencia hacia la situación de aprendizaje y las expectativas del propio aprendiz. Las variedades obligan a ratificar el modelo y pluralizarlo. Dentro de la variedad que se seleccione como preferente para un curso concreto, han de insertarse rasgos de las restantes variedades periféricas secuenciados en atención a su adecuación dentro del propio syllabus del curso y seleccionados según criterios objetivos.

En este trabajo hemos pretendido ofrecer una fórmula válida para la construcción de este modelo lingüístico de enseñanza del español que teóricamente recogiera todos los componentes que deben estar representados y que permitiera seleccionarlos con libertad en cada situación de enseñanza. Con especial interés, nos hemos detenido en las variedades por la complejidad que ellas ofrecen.

\section{Referencias bibliográficas}

Andión Herrero, M. ${ }^{\mathrm{a}}$ A. (en prensa): "El español como lengua extranjera: relación entre el estándar y las variedades". En Actas del X Congreso Brasileño de Profesores de Español, Natal. Brasil, Consejería de Cultura de la Embajada de España en Brasilia, 2003.

Andión Herrero, M. ${ }^{a}$ A. (2005): Las variedades del español en América: una lengua y 19 países. Apuntes para profesores de E/LE, Brasil, Consejería de Educación de la Embajada de España en Brasilia, Brasil, 2005.

Andión Herrero, M. ${ }^{a}$ A. (en prensa): "Modelo, estándar y norma..., conceptos imprescindibles en el español L2/LE", RESLA.

Beaven, T. y C. Garrido (2000): "El español tuyo, el mío, el de aquél... ¿Cuál para nuestros estudiantes?”. En Martín Zorraquino, M. ${ }^{a}$ A. y C. Díez Pelegrín (eds.), ¿Qué español enseñar?: Norma y variación lingüísticas en la enseñanza del español a extranjeros. Actas del XI Congreso Internacional ASELE, Zaragoza, págs. 181-190.

Blanco, C. (2000): "El dominio del concepto de norma como presupuesto del profesor de ELE?". En Martín Zorraquino, M. ${ }^{a}$ A. y C. Díez Pelegrín (eds.), ¿Qué español enseñar?: Norma y variación lingüísticas en la enseñanza del español a extranjeros. Actas del XI Congreso Internacional ASELE, Zaragoza, págs. 209-216. 
Blecua, J. M. (2001): "Unidad, variedad y enseñanza". En II Congreso Internacional de la Lengua Española. El español en la sociedad de la información, Instituto Cervantes, Madrid: http://cvc.cervantes.es/obref/congresos/valladolid/ponencias/unidad diversidad del espanol/1 la norma hispanica/blecua j.htm (25-12-2007).

Demonte Barreto, V. (20001): "El español estándar (ab)suelto. Algunos ejemplos del léxico y la gramática". En II Congreso Internacional de la Lengua Española. El español en la sociedad de la información, Instituto Cervantes, Madrid: http://cvc.cervantes.es/obref/congresos/valladolid/ponencias/unidad_diversidad_del_espanol/1_la norma hispanica/demonte v.htm (12-01-2008).

Gutiérrez Ordóñez, S. (2001): "Perfiles y dimensiones en el concepto de norma (las otras normas)". En II Congreso Internacional de la Lengua Española. El español en la Sociedad de la Información, Instituto Cervantes, Madrid: http://cvc.cervantes.es/obref/congresos/valladolid/ponencias/unidad_diversidad_del_espanol/1_la norma hispanica/gutierrez_s.htm (28-01-2008).

Instituto Cervantes (2006): Plan Curricular del Instituto Cervantes: niveles de referencia para el español. Madrid, Editorial Biblioteca Nueva, S. L., 3 tomos.

Lope Blanch, J. M. (1993): "La norma lingüística hispánica". En II Congreso Internacional de la Lengua Española. El español en la Sociedad de la Información, Madrid, Instituto Cervantes http://:cvc.cervantes.es/obref/congresos/valladolid/ponencias/unidad diversidad del espanol/1 1 a norma hispanica/lope i.htm\#5 (28-01-2008).

López García, Á. (1997): "La percepción del español como LE", Español Actual, 67, págs. 7-15.

López García, Á. (2000): "El contacto de lenguas y la singularidad americana". En Calvo Pérez, J. (ed.), Teoría y práctica del contacto: el español de América en el candelero, Frankfurt, Vervuert, págs. 17-31.

López Morales, H. (2006): "El futuro del español". En Instituto Cervantes, Enciclopedia del español en el mundo. Barcelona, EGEDSA, págs. 476-491.

Moreno Fernández, F. (1998): Principios de sociolingüística y sociología del lenguaje. Barcelona, Ariel.

Moreno Fernández, F. (2000): Qué español enseñar. Madrid, Arco/Libros.

Moreno Fernández, F. (2001): "Prototipos y modelos de lengua", Carabela. Modelos de uso de la lengua española, 50, págs. 5-20.

Narbona Jiménez, A. (2001): "Movimientos centrífugos y centrípetos en la(s) norma(s) del español". En II Congreso Internacional de la Lengua Española. El español en la Sociedad de la Información, Madrid, Instituto Cervantes: http://www.cvc.cervantes.es/obref/congresos/valladolid/ponencias/unidad diversidad del espan ol/1 la norma hispanica/narbona a.htm (28-01-2008).

Tejera, M. ${ }^{a}$ J. (2003): "La tercera norma del español de América. En Lengua, variación y contexto. Estudios dedicados a Humberto López Morales. Madrid, Arco/Libros, t. II, págs. 455-467.

Tibor, B. (2000): "Norma y uso en la clase de E/LE: Análisis de la norma académica y los libros de texto”. En Martín Zorraquino, M. a A. y C. Díez Pelegrín (eds.), ¿Qué español enseñar?: Norma y variación lingüísticas en la enseñanza del español a extranjeros. Actas del XI Congreso Internacional ASELE, Zaragoza, págs. 201-208. 